\title{
POLÍTICAS Y LUGARES DE LA MEMORIA. LA \\ RECONSTRUCCIÓN DEL PASADO EN EL PRESENTE. EL CASO \\ DEL ESPACIO MANSIÓN SERÉ EN BUENOS AIRES, ARGENTINA
}

POLICIES AND PLACES OF MEMORY.RECONSTRUCTION OF THE

PAST IN THE PRESENT.CASE STUDY OFMANSIÓNSERÉSPACE IN

BUENOS AIRES, ARGENTINA

Enviado em 8 de agosto de 2015

Aceito em 11 de agosto de 2015

Cora Escolar ${ }^{1}$

Silvina Fabri ${ }^{2}$

Resumen: Nos proponemos en este artículo reflexionar acerca de lo tendencialmente infinita que es la reconstrucción del pasado en el presente. De ahí, que en un primer momento, estimamos significativo hablar sobre los "lugares de memoria", para debatir la relación entre la memoria y el olvido. Esos lugares que, habitualmente, cumplen una función de simbolización, articulan nuevos sentidos y significaciones con el fin de conmemorar/rememorar el pasado reciente. Es en el caso de la ex Mansión Seré donde pensamos centrar nuestra atención. En este sentido, el espacio conmemorativo plantea la posibilidad de reflexionar sobre las políticas de la memoria, el hacer institucional y el modo en el que ambos se conjugan.

En un segundo momento, pretendemos sostener que la memoria se sustancia en recuerdos, es decir, en "re-acuerdos". Pensamos que construye un nosotros y un imaginario social que conforman horizontes de pertenencia e identidad.

Palabras clave: Políticas y lugares de la memoria. Reconstrucción del pasado. Memoria.

Abstract: This article reflectson how the reconstruction of the past in the present is an infinite process. Thus, firstly, it is considered significant to speak

\footnotetext{
1 Socióloga. Profesora Titular Regular e Investigadora del Departamento e Instituto de Geografía, Facultad de Filosofía y Letras, Universidad de Buenos Aires. Investigadora Categorizada Nivel I del Sistema Nacional de Investigación, Programa de Incentivos a la Investigación, Ministerio de Cultura y Educación. Directora del Proyecto UBACYT 127/BA: "Políticas, instituciones y saberes. La hechura de lugares de memoria (1955-2013)", período de programación: 2014-2017. Ejerce como docente de posgrado de Epistemología de las Ciencias Sociales y Metodología de la Investigación en doctorados, maestrías y especializaciones de la UNSAM, UNLa y UBA. E-mail: cescolar@fibertel.com.ar

${ }^{2}$ Geógrafa. Profesora e Investigadora del Departamento e Instituto de Geografía, Facultad de Filosofía y Letras, Universidad de Buenos Aires. Becaria Doctoral del Proyecto UBACYT 127/BA: "Políticas, instituciones y saberes. La hechura de lugares de memoria (1955-2013)", período de programación: 20142017.
} 
of" places of memory" within the debate regarding the relation between memory and forgetting. Those places of memory habitually have a symbolizing function, and also articulate new sentiments and meanings with the goal of commemorating and remembering the recent past. This article concentrates on the case of the ex-Mansión Seré. In this case the commemorative space gives the possibility to reflect on memory policies, institutional workings, and they way that both interact with each other.

Secondly, the article sustains that the substance of memory are memories, and that memory builds a collective "we" and a social imaginary that make up our horizons of identity and sense of belonging.

Keywords: Policies and places of memory. Reconstruction of the past. Memory.

\section{ALGUNAS PALABRAS INICIALES}

Aunque no a fondo, en el abstract planteamos cuán tendencialmente infinita es la reconstrucción del pasado en el presente. Esto nos lleva a pensar cuán inagotable es la memoria y al mismo tiempo nos permite percibir la limitación de lo vivido. La reconstrucción del pasado en el presente es capaz de resucitar a cada instante con distintos rostros y valencias. En ese presente que constituye la experiencia social somos las historias que podemos narrarnos a nosotros mismos. El pasado es siempre presente de pasado y el futuro presente de futuro. Es decir, por una parte, continua recreación del recuerdo y, por otra, una anticipación que la memoria convierte en imaginado recuerdo prospectivo.

A menudo se insinúa que todas las sociedades han mostrado un ilimitado interés por administrar la memoria social. La memoria se sustancia en recuerdos, es decir, en "re-acuerdos". La memoria es indispensable acervo que el transitar de los sujetos sociales transmite, urdimbre de su mundo interno. La memoria es un importante agente de configuración de sentido, capaz de articular intervalos en un presente permanentemente contextualizado y operativo a los fines de cada vivir. Los sujetos precisan recordar el ayer a fin de tomar conciencia de su pertenencia a una tradición y de abrirse a inéditas posibilidades de vida.

De pronto sentimos que el pasado es siempre nuevo. Cambia continuamente de la misma manera que la vida transcurre. Fragmentos del pasado que parecen que se han precipitado en el olvido irrumpen de nuevo, otros en cambio se difuminan más y más porque son -o creemos que son- menos importantes. Tenemos la sensación de que el presente dirige al pasado: así el pasado parece ahora largo, después corto. De pronto resuena, de pronto enmudece. 
De allí que nos atrevemos a sostener que el pasado es siempre "presente del pasado", continua creación del recuerdo, rescatado del olvido insondable. Y el futuro, "presente del futuro", una anticipación que la memoria convierte en imaginado recuerdo prospectivo.

\section{PENSAR ACERCA DE LA MEMORIA}

En este apartado trataremos de pensar sobre la memoria en cada acto conmemorativo que entendemos, expresa el nexo entre historia y razón, dado que quién recuerda lo hace a partir de sus criterios, posibilidades y preferencias. Después porque quién no puede recordar se disuelve en la fugacidad de los instantes vividos. "Somos las historias que podemos narrarnos a nosotros mismos", sean cuales fueren las coyunturas (Assmann, 2008, p. 135³)

Los pioneros estudios sobre la "memoria colectiva" escritos por Halbwachs (1994) otorgan una función socializadora a la memoria colectiva, ya que permite la ubicación de los sujetos y grupos en sus respectivos a horas. Se trata, para el autor mencionado de un fenómeno ante todo social, "el grupo se asegura de su pertenencia con la reconstrucción del pasado tanto como el individuo se asegura de su pertenencia al grupo con su recuerdo" (Halbwachs, 1994, p. 27)

$\mathrm{Y}$, así como los lugares de la memoria pueden tener un carácter gratificante y saludable (Tierra Santa, por ejemplo) también pueden poseer un carácter traumático e inquietante (Auschwitz, por ejemplo). De ahí que estimamos necesario acercarnos a los actuales "lugares de la memoria" y reflexionar al respecto.

\section{EMPLAZAMIENTO DE LOS LUGARES DE LA MEMORIA}

Esta acción hace referencia, en un primer nivel de análisis y, desde la perspectiva del análisis institucional, al espacio -establecimiento donde se ubican los lugares de memoria.

Si bien los lugares de memoria están emplazados en un lugar físico determinado, sus funciones y extensión no están delimitadas por ese espacio físico. De allí que hablamos de área de influencia del lugar de la memoria. Área que no puede ser delimitada a priori sino que su alcance habrá de depender del interjuego que se produzca entre los actores sociales y de las condiciones que desde la comunidad y

$3 \mathrm{~J}$. Assman se refiere en concreto a la desaparición de los supervivientes de los campos de exterminio nazis $(2008$, p. 135). 
desde el propio lugar sean generadas para el desarrollo de un trabajo memorial efectivo. Se trata de un juego permanente entre lo instituyente y lo instituido en el que las fronteras son concebidas como dinámicas y por lo tanto móviles.

Estas relaciones institucionales se convierten en fuerzas en movimiento que trascienden el espacio - físico, es decir el "lugar" de emplazamiento para extenderse y retroalimentarse con ese otro espacio que hemos definido como su área de influencia.

Es en ese sentido que las distintas prácticas hablan de la articulación y de ese movimiento dado por el "juego" permanente de lo instituido y lo instituyente, teniendo en cuenta que no estamos hablando de actividad y pasividad sino de dimensiones que realizan o des-realizan el complejo institucional. Estamos pensando en un nudo de relaciones complejas y móviles ${ }^{4}$ (Escolar, 2008, p. 183).

\section{RELACIONES CONCEPTUALES PARA PENSAR EL RE-}

\section{EMPLAZAMIENTO DE LA MANSIÓN SERÉ COMO LUGAR DE LA MEMORIA}

A partir de la década de 2000 un conjunto de procesos político-institucionales posibilitaron la recuperación de los espacios materiales que funcionaron como Centros Clandestinos de Detención (CCD) en la última dictadura militar en Argentina (19761983). Estos espacios fueron a partir de ese momento definidos como sitios de memorias ( $\mathrm{DDHH}, 2012)$. En ese contexto el predio en donde se encontraba la Mansión Seré (CCD Atila desde 1977 a 1978) se reconfigura y se resignifica ${ }^{5}$. Con el reemplazamiento ${ }^{6}$ de este sitio como un lugar de la memoria - su reinscripción en la trama urbana sobre la base de nuevos objetivos y operando desde la construcción de una narrativa particular - se denuncian y visibilizan los usos del predio en el pasado reciente $\mathrm{y}$, a partir de ello, se lleva adelante una tarea reflexiva en torno a la construcción de la memoria social (Halbwachs, 1994).

Nos preguntamos cómo conceptualizar este sitio como un lugar de la memoria y cómo se produce la espacialización del recuerdo; cómo el recuerdo traza conexiones

\footnotetext{
${ }^{4}$ Escolar (2008, p.183) escribía sobre los observatorios de derechos humanos que en el sentido que tiene este párrafo se acerca a nuestra perspectiva sobre los lugares de memoria.

${ }^{5}$ El predio funcionó desde el año 1985 como Polideportivo Municipal Gorki Grana. En el año 2000 con la inauguración de La Casa de La Memoria y la Vida, sede de la Dirección de Derechos Humanos del Municipio de Morón, se inician las tareas para recuperar los restos materiales de la Mansión Seré. En el año 2013 se inaugura, formalmente, el Espacio Mansión Seré (EMS) como un Centro de Investigación e Interpretación donde se realizan las visitas guiadas por las excavaciones arqueológicas de los cimientos de la casona, incendiada en el año 1978 y finalmente demolida, en el período democrático, para instalar sobre ella una cancha de futbol (DDHH, 2012).

${ }^{6}$ A los efectos de este artículo queremos dejar aclarado que utilizamos la categoría de re-emplazamiento, para el caso de este sitio, subsumiendo a su interior el concepto de recuperación.
} 
y lazos con el espacio físico material y es reelaborado a partir de nuevas tramas de acciones y sentidos que se articulan y rearticulan dentro y a partir de transitar por el predio.

El lugar de la memoria como intervención político-institucional en el continum urbano pensado como una disrupción y una marcación en ese espacio se refiere a un pasado que pretende restituirse desde el presente y reelabora una relación entre el espacio, la memoria y las prácticas sociales. Esta sería una entrada posible para pensar los lugares de la memoria como sitios que nos interpelan desde el pasado, que nos hacen pensar la historia como algo no resuelto y permite releerla desde un contexto político-social trazado en el presente. La memoria es siempre actual y su actualización depende, en cierta medida, de los marcos sociales de la memoria (Halbwachs, 1994) de su contexto de emplazamiento.

Si tenemos en cuenta que la ciudad de hoy anuda sus sentidos y significados con la ciudad de ayer, podemos pensar en un espectáculo que

preserva y restaura, decapa sus fachadas, monumentaliza y patrimonializa la ciudad pasada, a la que al mismo tiempo reconstruye (...) la ciudad se observa, se busca y se fuga al mismo tiempo en sus anales y sus estratos aplastados unos contra otros (Nancy, 2012, p.38).

Estos solapamientos y rearticulaciones que estructuran la ciudad y lo urbano nos presentan a los lugares de la memoria como nexos construidos entre eso que ya pasó y su significación actualizada constantemente. La ciudad se transforma de manera incesante de "real" a paisaje simbólico, es reanimada y potenciada (Berman, 1988) por mecanismos memoriales que instauran una forma novedosa de hacerse con ella (Nora, 1998).

Hablar de lugares de la memoria supone entonces tomar ciertos recaudos y precauciones pues no son meros elementos materiales como podrían ser los monumentos puramente físicos, palpables y visibles ni acontecimientos pasibles de ser memorables, que contienen por sí mismos la capacidad de dar un sentido memorial del pasado. La idea avanza hacia una dimensión más compleja que anuda

una noción abstracta, puramente simbólica, destinada a desentrañar la dimensión rememorada de los objetos que pueden ser materiales, pero sobre todo inmateriales (...) Se trata de comprender la administración general del pasado en el presente, mediante la disección de sus polos de fijación más significativos (Nora, 1998, p. 32).

El concepto de emplazamiento se encuentra asociado a colocar, situar y posicionar un objeto en un lugar: la acción de emplazar equivale a trazar un ámbito de 
acción para determinado objeto. Para Corominas (1973, p.463) proviene de "Plazo, del arcaico plazdo. Deriv. Aplazar. Emplazar., emplazado, emplazamiento". Según Moliner (2007, p. 1133) el mismo término proviene de "(en- y plazo). Poner una cosa en el sitio donde ha de funcionar. Situar".

Recuperando las reflexiones hasta aquí desarrolladas podemos relacionar estas definiciones con los presupuestos que se desprenden de la perspectiva del giro cultural en geografía. La misma plantea el estudio y la problematización de los elementos culturales que intervienen en las conformaciones de los lugares en escalas de significación particular (Lindón, 2011). En este sentido, el re-emplazamiento potencia la relación entre el espacio físico y material con los espacios construidos subjetivamente en relación a cambios contextuales, particulares y específicos de un momento dado.

Imagen I: Espacio Mansión Seré (Centro de interpretación) donde se realiza el recorrido a través de pasarelas por los cimientos de la casona Mansión Seré, foco del trabajo arqueológico de recuperación iniciado en el año 2000. Allí, con el marco de los días conmemorativos se llevan adelante intervenciones artísticas como obras de teatro, muestras fotográficas y conciertos musicales.

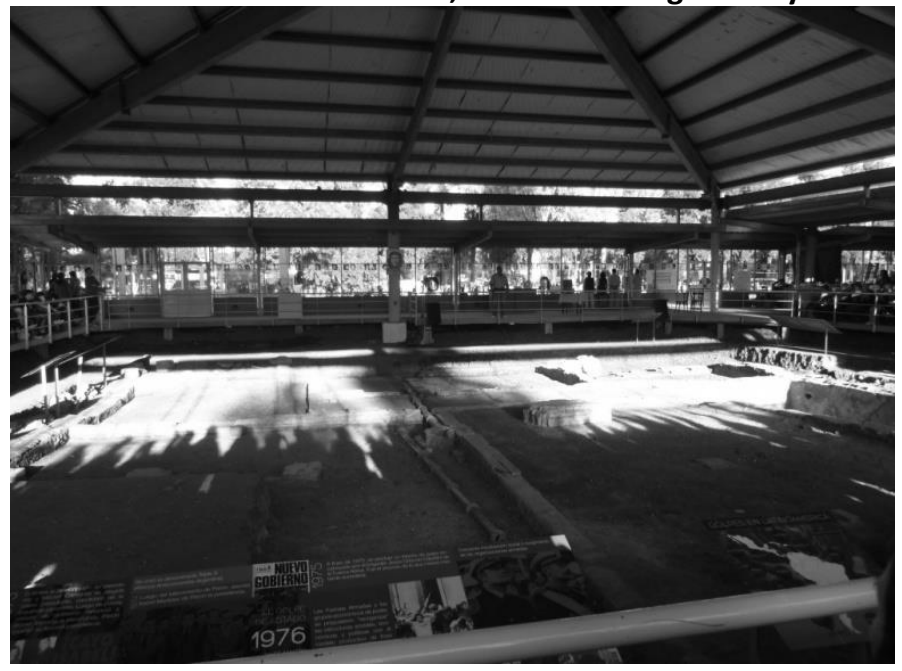

Fuente: Fotografía tomada por Silvina Fabri el día 23 de marzo de 2014 con motivo del 38 Aniversario del Golpe de Estado de 1976.

Siguiendo este planteo las Geografía Culturales han ocupado en los últimos años un lugar destacado en la disciplina mostrándose como un ámbito de trabajo inter y transdisciplinario. Ese diálogo fluido con otros campos y perspectivas posibilitan la construcción de nuevas aproximaciones a problemáticas variadas de las cuales, a los fines de este escrito, nos interesan: las expresiones culturales que imprimen valor simbólico a los lugares. En palabras de Lindón y Hierneaux 
el giro cultural planteó el redescubrimiento de la dimensión cultural en geografía y su presencia, de una forma u otra, en casi todos los campos de la disciplina. (...) Lo que estamos planteando es la construcción de un nuevo edificio teórico en torno al concepto de espacio $(2006$, p.9)

Continuando el planteo de Lindón sobre la posibilidad de otorgar inteligibilidad a las prácticas socio-espaciales, los fragmentos espaciales como los lugares cobran significación y relevancia para los análisis que pretendan incorporar la construcción subjetiva de los lugares. Los lugares de la memoria estarían atravesados por estas implicaciones, podríamos pensarlos como espacios vividos en los

cuales los sujetos-habitantes realizan prácticas con implicaciones espaciales, haciendo uso de saberes y competencias territoriales, recurriendo a su memoria espacial, realizando negociaciones con los otros respecto al uso y apropiación del lugar, aun cuando se trate de formas de apropiación efímeras (2011, p.203204).

Como sostiene Bozzano (2009) no sólo opera en nuestra vida cotidiana el espacio cartesiano o euclidiano que soporta medidas, distancias geométricas espaciales o reglas matemáticas que rigen los espacios entendidos como absolutos o relativos y muestran a las prácticas espaciales ${ }^{7}$ a partir de su mensurabilidad sino que "en nuestras percepciones, sentidos, emociones, valoraciones y acciones, está arraigada otra concepción espacial, menos difundida que aquella: se trata del espacio topológico" (2009, p.159.Resaltados en el original). De esta manera, este espacio puede tratar aspectos no mensurables, aspectos más bien interpretables de muy diversa manera mediante posiciones de atributos tangibles e intangibles reconocibles en sujetos y en objetos o en las relaciones entre ambos.

La interacción constante entre subjetividades emergentes (Vargas Isla y Fernández, 1994) y las relaciones que estas establecen con el medio que transitan (Nancy, 2012), consumen y vivencian cotidianamente (De Certeau, 1974) pueden encarnar símbolos compartidos que asociados a la memoria colectiva y a la conformación de lugares de la memoria requieren de ciertas especificaciones, particularidades y elementos que conllevan a que un lugar se convierta en lugar de la memoria (Nardi, 2009). Por ello,

\footnotetext{
7 La vida cotidiana de los sujetos en relación a la construcción de lugares (Lindón, 2000; 2007) se enlaza, al mismo tiempo, con las estrategias que establecen de qué manera se fabrica una ciudad, es decir, la materialidad de las políticas de intervención urbanística, con sus códigos y sus reglas de convivencia, los marcos legales o morales sobre las que se vigilan los comportamientos sociales. De esta manera, se reelabora una red de relaciones infinitamente amplia habilitando formas de apropiación diferenciales y prácticas sociales en constante cambio (De Certeau, 1974).
} 
lo que antes era un mero 'espacio' físico o geográfico se transforma en 'lugar' con significados particulares, cargados de sentidos y sentimientos para los sujetos que los vivieron (Jelin y Langland, 2003, p.3. Resaltados en el original).

Pero, al mismo tiempo, el espacio se reconfigura para quienes hoy utilizan el predio en el desarrollo de sus actividades cotidianas y lo hacen propio estableciendo lazos simbólicos, culturales o políticos con el lugar (Arfuch, 2005).

Imagen II: La Casa de la Memoria y La Vida, sede de la Dirección de Derechos Humanos del Municipio de Morón con motivo de las actividades conmemorativas del 38ㅇ Aniversario del Golpe de Estado de 1976.

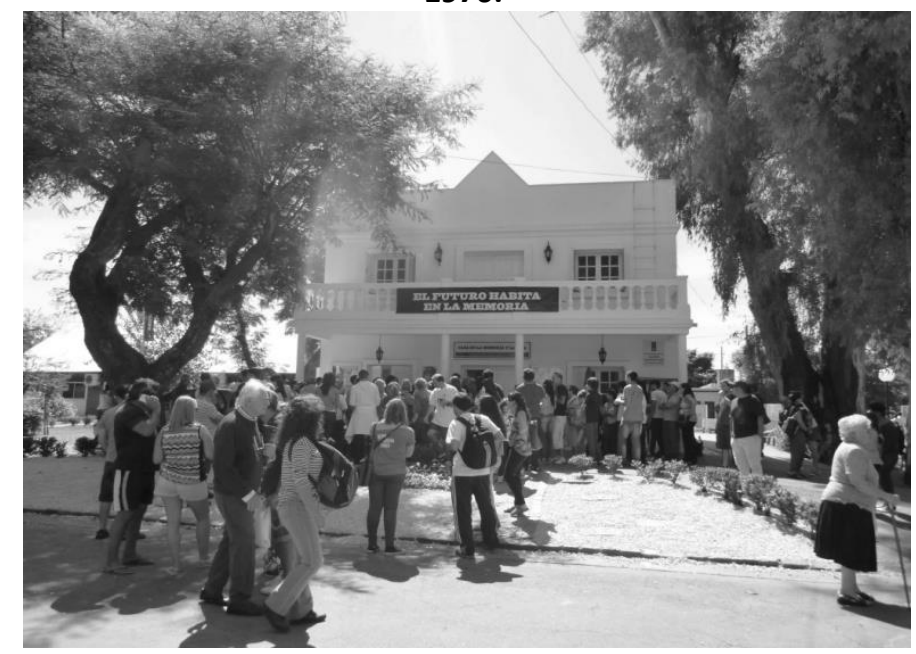

Fuente: Fotografía tomada por Silvina Fabri, martes 24 de marzo de 2014.

Así, el Espacio Mansión Seré puede servir como ejemplo de este recorrido puesto que el lugar estuvo siempre allí pero el lugar de la memoria necesitó de ciertas tramas institucionales para reconfigurarse y narrar nuevos sentidos (Fabri, 2011). Las políticas de memoria se anudan con el espacio socialmente construido conformando un proceso complejo y generando efectos de lugarización de la memoria. Por lo tanto

toda política pública es el producto del enfrentamiento y la negociación entre diferentes proyectos, los que a su vez emergen de diferentes actores sociales. En tal sentido, si interpretamos a las políticas públicas como resultado de estas negociaciones sociales, podemos considerarlas como indicadores de la distribución del poder dentro del mismo estado como así también de la sociedad (Escolar, Besse y Lourido, 1994, p. 137).

De esta manera, el concepto de re-emplazamiento puede contribuir a dilucidar y repensar las diversas prácticas sociales que se dan al interior del proceso de construcción de un lugar de la memoria e intentar conectar los conceptos de espacio, lugar y territorio con las prácticas diferenciales a las que cada uno hace referencia (Fabri, 2012). 
Así, el re-emplazamiento nos abre la posibilidad de reflexionar sobre el alcance del término recuperación utilizado para hablar de cómo los ex CCD son tratados una vez que determinada política pública de memoria se implementa para reconstruir ese espacio cargándolo de nuevos significados. Puesto que como sostiene Feld (2011) la recuperación no implica recobrar algo que se poseía previamente sino que puede considerarse como una categoría nativa utilizada por los diversos emprendedores de la memoria para referirse al modo en que ese proceso se ha desarrollado. Por ello, "se entiende que hay una mutua implicación entre las categorías de actor social, acción social y espacio social, que no pueden pensarse de manera autónoma, aislada cada una de las otras" (Escolar, 2008, p. 184).

Como afirman Escolar y Palacios,

la memoria necesita encarnarse espacialmente para seguir testimoniando y este proceso coadyuva en la configuración de ese espacio material y en cómo ese espacio es incorporado e integrado a la vida cotidiana a partir de la implementación de la política pública de la memoria $(2010$, p.8).

La implementación y la gestión de las políticas que llevan a lugarizar la memoria trazan un marco de referencia delineado a través de la capacidad de decisión de los actores intervinientes (Jelin, 2002). Pensamos, en este sentido, que es a partir del proceso de reapropiación del espacio material que el sitio de memoria puede constituirse como tal, la apropiación simbólica anuda la resignificación de este espacio (Trotta, 2009), los relatos que de él se construyen y los trabajos de memoria que de él se desprenden.

Imagen III: En la entrada de La Casa de la Memoria y La Vida, enmarcando el acceso a la DDHH, la intervención urbana Huellas de fuego, homenaje al camino recorrido en torno al reclamo de Memoria, Verdad y Justicia de madres y padres de detenidos desaparecidos en la última dictadura militar en Argentina.

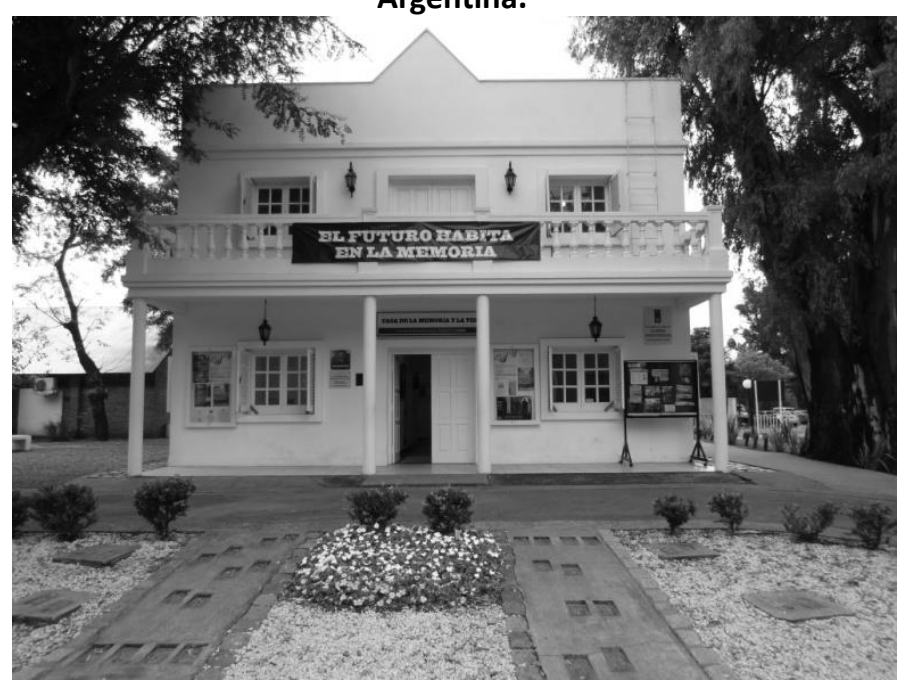

Fuente: Fotografía tomada por Silvina Fabri el día 30 de mayo de 2014. 


\section{CONCLUSIONES. LAS FRONTERAS DEL TIEMPO}

Qué hacemos cuando recordamos? Tal vez lo que hacemos es fundar el pasado, es decir, establecer unas particulares y específicas fronteras del tiempo. El pasado no está ahí, con sus reclamaciones intactas, esperando ser atendidas sino que es la materia prima de un determinado quehacer histórico... la tarea de la memoria (Ricœur, 2003).

Decía Hannah Arendt (1993, p.22) que la acción humana desarrolla consecuencias hasta el infinito. Podríamos añadir que en una doble dirección: tanto hacia el futuro como hacia el pasado. No recordamos porque en algún momento actuamos, sino que actuamos para poder recordar.

\section{BIBLIOGRAFÍA CONSULTADA}

ARENDT, Hannah. La condición humana. Barcelona: Paidós,1993.

ARFUCH, Leonor (comp.). Identidades, sujetos y subjetividades. Buenos Aires: Prometeo Libros, 2005.

ASSMAN, Jan. Religión y memoria cultural: diez estudios. Buenos Aires: Libros de la Araucaria, 2008.

BERMAN, Marshal. Todo lo sólido se desvanece en el aire: La experiencia de la modernidad. México: Siglo XXI, 1988.

BOZZANO, Horacio. Territorios posibles. Procesos, lugares y actores. Buenos Aires: Ed. Lumiere, 2009

COROMINAS, Joan. Breve Diccionario Etimológico de la Lengua Castellana. Madrid: Ed. GREDOS, 1973.

DE CERTEAU, Michel. Conclusión: De los espacios y de las prácticas. En: La cultura en plural. Buenos Aires. Nueva Visión, págs. 189-204, 1974.

ESCOLAR, Cora. Memoria y vida. Reflexiones epistemológicas acerca del discurso institucionalizado de la memoria. En: ACCIONES, Investigaciones Sociales. Universidad de Zaragoza, págs. 55-68, 2009. 
ESCOLAR, Cora. Del compromiso político a la lucha por los derechos. Los Observatorios de Derechos Humanos como enclave territorial. En: Geousp - Espaço e Tempo. Revista da Pósgraduação em Geografia. Número 24. Brasil. Universidad de São Paulo, págs. 182-187, 2008.

ESCOLAR, Cora; Juan Besse y Clara Lourido. Redes para 'pescar' lo real (un abordaje teóricometodológico). En: Revista Realidad Económica. № 124. Buenos Aires: IADE, págs. 135-144, 1994.

ESCOLAR, Cora y Cecilia Palacios. La producción del espacio urbano y la dimensión espacial de las prácticas institucionales. El caso del Espacio para La Memoria y Para la Promoción y defensa de los Derechos Humanos (Ex ESMA). En: XI Coloquio internacional de Geocrítica. Buenos Aires, 2010. Disponible en: www.filo.uba.ar/contenidos/investigacion/.../geo/geocritica 2010/01.htm

HALBWACHS, Maurice. Los marcos sociales de la memoria. Barcelona: Anthropos, 1994. FABRI, Silvina. Los lugares de la memoria. Mansión Seré a diez años de su recuperación. En: Geousp - Espaço e Tempo. Revista da Pós-graduação em Geografia. Brasil. São Paulo. № 29, págs. 169-183, 2011.

FABRI, Silvina. Memoria, instituciones y espacio urbano. El re -emplazamiento de un sitio de la memoria. En: III Jornadas Internacionales de Problemas Latinoamericanos "Movimientos Sociales, Estados y Partidos Políticos en América Latina: (re)configuraciones institucionales, experiencias de organización y resistencia". Facultad de Ciencias Políticas y Sociales. Mendoza. Argentina: Universidad Nacional de Cuyo, 2012.

FELD, Claudia. Prólogo. La memoria en su territorio. En: Fleury, B. y W. Jacques (comps): Memorias de la piedra. Ensayos en torno a lugares de detención y masacre. Buenos Aires: Ed. Ejercitar la Memoria, págs. 9-17, 2011.

JELIN, Elizabeth. Los trabajos de la memoria. Madrid: Siglo XXI/SSRC, 2002.

JELIN, Elizabeth y Victoria Langland (comps.). Monumentos, memoriales y marcas territoriales. Madrid: Siglo XXI/SSRC, 2003.

LINDÓN, Alicia. La vida cotidiana y su espacio-temporalidad. Madrid: Anthropos. 2000. LINDÓN, Alicia. Los imaginarios urbanos y el constructivismo geográfico: los hologramas espciales. En: EURE, XXXIII, v.99, págs. 31-46, 2007.

LINDÓN, Alicia. Revisitar la concepción de lo social para una Geografía constructivista. En: Zusman, Perla, R. Haesbaert, H. Castro y S. Adamo (ed.). Geografías Culturales. Aproximaciones, 
intersecciones y desafíos. Buenos Aires: Ed. FFYL, Universidad de Buenos Aires, págs. 177-212, 2011.

LINDÓN, Alicia y Daniel Hierneaux . Introducción. La geografía humana: un camino a recorrer. En: Tratado de Geografía Humana. México - Iztapalapa: Ed. Anthropos. Universidad Autónoma Metropolitana, págs. 7-22, 2006.

MOLINER, María. Diccionario de uso del español. Madrid: Ed. GREDOS, 2003.

NANCY, Jean. La ciudad a lo lejos. Buenos Aires: Manantial, 2012.

NARDI, Silvia. Las paredes de la memoria. Recuerdos, registros y reflejos de una sociedad. En: AAVV. Los lugares de la memoria. Buenos Aires: Ed. Madreselva, págs. 11-26, 2009.

NORA, Pierre. La aventura de Les lieux de mémoire. Memoria e Historia. En: Josefina Cuesta Bustillo (ed): Revista Ayer, № 32, págs. 17-34. Madrid: Marcial Pons. 1998.

TROTTA, Andrea. Sitios de memoria: Espacios de apropiación simbólica. Relación del pueblo con su pasado y el papel del artista en la resignificación de los espacios públicos. En: AAVV. Los lugares de la memoria. Buenos Aires: Ed. Madreselva, págs. 41-56, 2009.

RICCEUR, Paul. La memoria, la historia, el olvido. Madrid: Fondo de Cultura Económica, 2003. VARGAS ISLA, Lidia y Lilia Esther Fernández Rivas. ¿Sujeto social o subjetividades emergentes?. En: Revista Tramas, № 6, junio, págs. 81-101, 1994, disponible en: http://tramas.xoc.uam.mx /busqueda.php?indice=AUTOR\&terminos=Fern\%C3\%A1ndez\%20Rivas,\%20Lidia\&indice_resulta dos $=0$ \&pagina $=1$.

\section{FUENTES DE INFORMACIÓN CONSULTADAS}

DIRECCIÓN DE DERECHOS HUMANOS (DDHH) (2012). Casa de la Memoria y La Vida. Su historia y sus protagonistas. Buenos Aires. Ed. Municipio de Morón. 